\title{
Eigenvalue extraction from time domain computations
}

\author{
T. Banova ${ }^{1,2}$, W. Ackermann ${ }^{2}$, and T. Weiland ${ }^{2}$ \\ ${ }^{1}$ Technische Universität Darmstadt, Graduate School of Computational Engineering, Dolivostraße 15, 64293 Darmstadt, \\ Germany \\ ${ }^{2}$ Technische Universität Darmstadt, Institut für Theorie Elektromagnetischer Felder (TEMF), Schlossgartenstraße 8, \\ 64289 Darmstadt, Germany
}

Correspondence to: T. Banova (banova@gsc.tu-darmstadt.de)

\begin{abstract}
In this paper we address a fast approach for an accurate eigenfrequency extraction, taken into consideration the evaluated electric field computations in time domain of a superconducting resonant structure. Upon excitation of the cavity, the electric field intensity is recorded at different detection probes inside the cavity. Thereafter, we perform Fourier analysis of the recorded signals and by means of fitting techniques with the theoretical cavity response model (in support of the applied excitation) we extract the requested eigenfrequencies by finding the optimal model parameters in least square sense. The major challenges posed by our work are: first, the ability of the approach to tackle the large scale eigenvalue problem and second, the capability to extract many, i.e. order of thousands, eigenfrequencies for the considered cavity. At this point, we demonstrate that the proposed approach is able to extract many eigenfrequencies of a closed resonator in a relatively short time. In addition to the need to ensure a high precision of the calculated eigenfrequencies, we compare them side by side with the reference data available from CEM3D eigenmode solver. Furthermore, the simulations have shown high accuracy of this technique and good agreement with the reference data. Finally, all of the results indicate that the suggested technique can be used for precise extraction of many eigenfrequencies based on time domain field computations.
\end{abstract}

\section{Introduction}

The field of quantum chaos encompasses the study of the manifestations of classical chaos in the properties of the corresponding quantum or more generally, wave-dynamical system (nuclei, atoms, quantum dots, and electromagnetic or acoustic resonators). Prototypes are billiards of arbitrary shape. In its interior a point-like particle moves freely and is reflected specularly at the boundaries. Depending on the shape its properties could exhibit chaotic dynamics.

Within this work, we investigate quantum billiards with its statistical eigenvalue properties, which reveal the periodic orbits in the quantum spectra and give the quantum chaotic scattering (Dembowski et al., 2005). Specifically, we simulate microwave resonator with chaotic characteristics, see Fig. 1, and we compute the eigenfrequencies that are needed for its level spacing analysis (Dembowski et al., 2002). Accordingly, the eigenfrequency level spacing analysis for determining the statistical properties requires many (in order of thousands) eigenfrequencies to be calculated and the accurate determination of the eigenfrequencies has a crucial significance. Moreover, considering that the problem is to compute a large number of eigenfrequencies, they can be often located in different ranges, i.e. left-most, right-most or interior portions of the spectrum could be sought.

In many scientific and engineering applications, as well as, in computational science, this results in solving one of the fundamental problems, the large scale eigenvalue problem. We list below just a few of the applications areas (Saad, 2011) where eigenvalue calculations arise: acceleration of charged particles, structural dynamics, electrical networks, Markov chain techniques, combustion processes, chemical reactions, macro-economics, control theory, etc. The above-mentioned realistic applications frequently challenge the limit of both computer hardware and numerical algorithms, as the involved matrices are of large scale.

The frequency domain methods (Jacobi-Davidson, Arnoldi, Lanczos, Krylov-Schur etc.) for eigenvalue calculation for some cavity structures might result in an extremely time consuming simulation, along with slow convergence and huge storage space. Particularly, structures with complicated geometry require a large number of grid points to achieve accurate simulation results. An additional 
challenge from our application is that the dimension of the desired eigen subspace is large, namely, one might possibly need thousands of eigen pairs located in a specified range, also referred to as a "window", of matrices with dimension in excess of several millions. Despite the fact that many algorithms for eigenvalue determination exist, accompanied by the numerical models that are becoming increasingly more sophisticated, not as many are specifically adapted for computing a large number of interior eigen pairs. They can usually calculate limited number of extreme or interior eigenfrequencies, and relatively few are designed for effectively reusing a large number of good initial presumptions, when they are available. Finally, computing a large number of interior eigenvalues remains one of the most difficult problems in computational linear algebra today.

In this context, our investigations comprise efficient, robust and accurate computations of many desired eigenfrequencies in a reasonable time, which also constitutes the main aim of our study. Within this work, we cover an approach for extraction of resonant frequencies given the output from time domain computations of closed resonators. The proposed approach uses the advantage that one single time domain simulation can provide the whole response of an electromagnetic system in a wide frequency band, whereas a frequency domain formulation uses one computation for each individual frequency. In addition, due to the fact that the time domain computations in the field of electromagnetics are already highly developed and considerably more efficient, as well as the fact that the transient solver contained in CST Microwave Studio (CST, 2012) uses a high degree of parallelization provided with modern graphics processing units (GPUs) feature the simulation can be dramatically accelerated. Therefore, we can easily and quickly get the time domain responses for a wide frequency band. In this way, significant reduction in computation time can be achieved and therefore, our high interest leads to time domain computations for electromagnetic problems.

The paper proceeds as follows. In Sect. 2 we briefly present the proposed approach for high precision eigenfrequency extraction given the available electric field computations. Here, we describe the fundamental modeling of the analyzed structure, followed by a description of the used signal postprocessing techniques. Section 3 investigates the simulation scenarios together with the obtained results. Lastly, conclusions are addressed in Sect. 4.

\section{Time domain approach for eigenfrequency extraction}

This section provides a brief overview of a precise timedomain (TD) approach for eigenfrequency extraction, which is applicable for different cavity structures. In a two-step process the modeling and simulation of a specific cavity structure is initially done, and afterward a postprocessing of the acquired time domain simulation results in MATLAB (MAT-

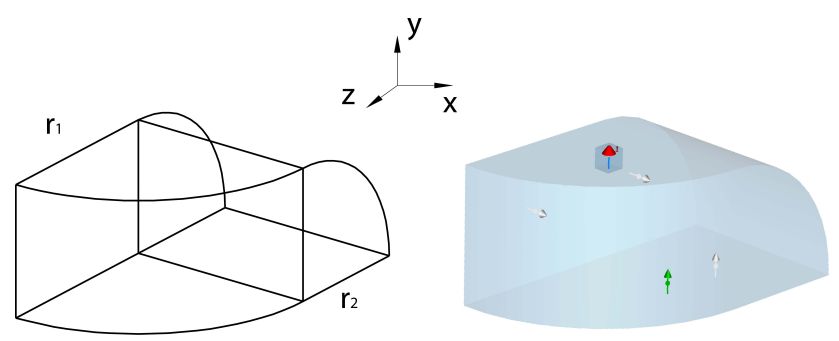

Fig. 1. Left: desymmetrized version of the three-dimensional generalized stadium billiard, consisted of two quarter cylinders with radii $r_{1}=200.0 \mathrm{~mm}$ and $r_{2}=141.4 \mathrm{~mm}$. Right: CST setup of the billiard cavity with an exciting antenna and detection probes placed at various positions.

LAB, 2011) is conducted. A descriptive sketch of the proposed TD approach is given in Algorithm 1. Additionally, the critical implementation points and details are covered, as well as, discussed within this section.

\subsection{Field simulation in time domain}

The proposed approach for eigenfrequency extraction (given in the following) is utilized within the project for determining the statistical properties of a cavity with chaotic characteristics. The requirements for chaotic characteristics are met by using a superconducting resonator with the shape of a desymmetrized three-dimensional stadium billiard (see Fig. 1-left). The billiard consists of two quarter cylinders with radii $r_{1}=200.0 \mathrm{~mm}$ and $r_{2}=141.4 \mathrm{~mm}$, respectively, and is made of niobium, which becomes superconducting at temperatures below $9.2 \mathrm{~K}$.

The cavity of interest is modeled in CST Microwave Studio (CST MWS) and a small tiny exciting antenna (as used in a physical model) is put properly in a way that the modes within a specific frequency range would be excited (see Fig. 1-right). Intentionally, the excitation signal applied at the antenna input is wide band signal, i.e. a Gaussian-modulated sinusoidal signal is chosen, which certainly covers the range of eigenfrequencies being sought. The field simulation with hexahedron discretization mesh in time domain is carried out with the transient solver from CST MWS and it detects and records the electric field intensity at specific field detection probes placed at various positions inside the cavity (see lines 1-6 in Algorithm 1). Later, the obtained time domain signals are used for further postprocessing in MATLAB, based on fitting techniques with a proposed model of the cavity response, as follows below.

\subsection{Postprocessing of the CST signals}

\subsubsection{Limitations from finite simulation time}

In view of the fact that we are dealing with superconducting walls, the response of a cavity could stay for a long time as 

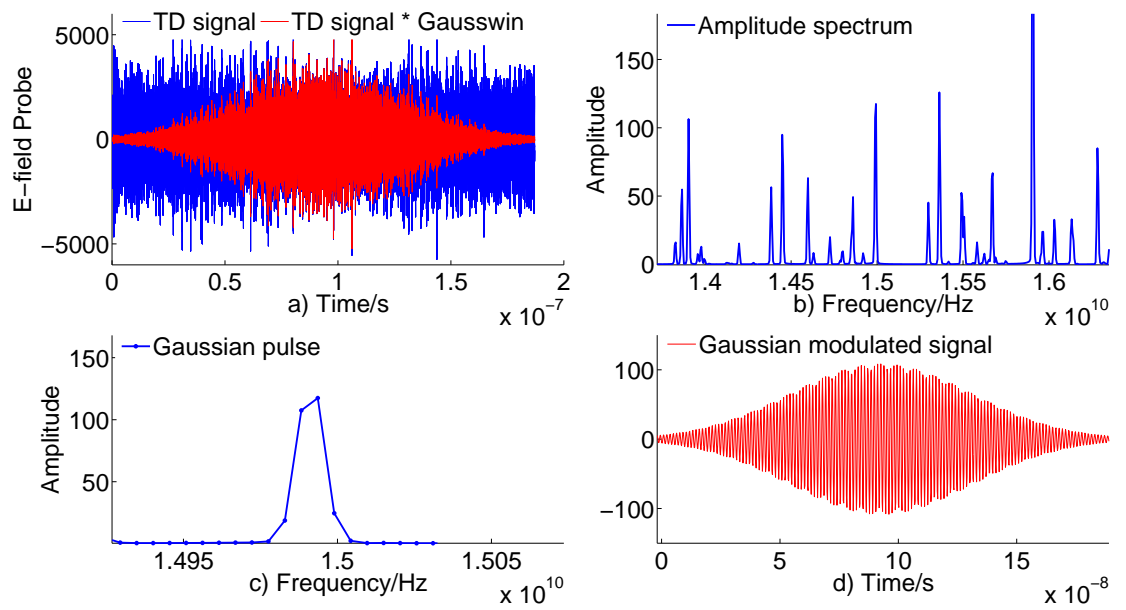

Fig. 2. Operating signals during the process of eigenfrequency extraction, listed in the respective sub-figures. (a) Acquired time domain signal and Gaussian windowed time domain signal. (b) Amplitude spectrum of the Gaussian windowed time domain signal. (c) Isolated Gaussian pulse within the eigenmode spectrum. (d) Gaussian modulated signal.

Input: Given a cavity with a specified probe $\mathrm{P}(\mathrm{x}, \mathrm{y}, \mathrm{z})$.

/* CST simulation */

Data: run transient simulation with the predefined simulation time.

Data: export a $T D \_s i g$ for the electric field intensity in $\mathrm{P}(\mathrm{x}, \mathrm{y}, \mathrm{z})$.

/* Post processing and frequency extraction in MATLAB

$T D \_s i g=T D \_s i g *$ gausswin $\left(\operatorname{size}\left(T D \_s i g\right)\right)$;

$f f t_{-} d a t a=f f t\left(T D \_s i g\right)$;

$a m p \_s p e c=\operatorname{abs}\left(f f t_{-} d a t a\right)$;

forall the Gaussian pulses in amp_spec do gauss_pulse = locate_gauss_pulse();

/ * ind_start=starting index ind_end=ending index

GoF $1=$ cftool(gauss_pulse,gauss_model ()$)$;

/ $* \mathrm{GoF}=$ Goodness of Fitting

if $G o F 1 \succ 0$ then

gausswin_fft_data $($ ind_start $:$ ind_end $)=$ $f f t \_d a t a *$ gausswin $($ ind_end-ind_start $)$; forall the $i n d \notin$ gauss_pulse do $\mid$ gausswin_fft_data $($ ind $)=0$; end gauss_mod_sig $=$ real(ifft $($ gausswin_fft_data $))$; GoF $2=$ cftool(gauss_mod_sig,gauss_mod_model()); if $G o F 2 \simeq 100$ then I extract the eigenfrequency; end

end

Algorithm 1: A sketch of the time domain approach for extracting eigenfrequencies. the power losses in the walls are negligible. Theoretically, the response of an ideal-conducting cavity is Dirac impulse sequence in frequency domain, i.e. a summation of sinusoidal signals with the associated eigenfrequencies in time domain. Nevertheless, due to the limited simulated time interval the ideal Dirac impulses of the true spectrum are widened. Moreover, due to the finite conductivity and the inserted antenna, the amplitude spectrum of the signal does not contain Dirac delta pulses, but it has pulses of finite width.

An important issue coming from the limitation in time is the discontinuities at the edges of the measured time (Mandal and Asif, 2010). Given that sharp discontinuities have broad frequency spectra, these will lead to a higher side lobes level and each spectral line of the signal's frequency spectrum will be spread out in the same way. In other words, the spreading means that signal energy, which should be concentrated only at one eigenfrequency, leaks instead into other frequencies, the so called "spectral leakage". Consequently, the whole spectrum is distorted and some weak impulses, i.e., eigenfrequencies, can be masked by the resulting convolution with neighboring strong pulses. This leads to the idea of multiplying the original signal within the measurement time by Gaussian function (see Fig. 2a) that smoothly reduces the signal to zero at the end points of the measurement time: therefore avoiding discontinuities overall (see line 9 in Algorithm 1). As a result, in case of an ideal cavity, whose spectrum theoretically is constituted of Dirac impulses located at the eigenfrequencies, we would have Gaussian pulses instead (see Fig. 2b).

Furthermore, manifestation of a spectral distortion occurs as result of a reduced spectral resolution. Namely, this is an important issue, and the minimum separation needed between two frequency components must be determined, so 
that they can be resolved. In our approach, we choose the frequency resolution good enough to recover the sought frequency data. As a result, a large number of simulation time samples might be needed for a reliable characterization of the resonance frequency of the structure, given that better frequency resolution unescapably requires a longer simulation time. In spite of this, the modern GPUs feature a large number of processing cores and the simulation is speeded up significantly in comparison with a simulation running on single CPU.

\subsubsection{Fast Fourier transformation}

As soon as the limitations that come from finite simulation time or reduced frequency resolution are overcame, the step from the recorded time domain response to the frequency domain response is computed using the fast Fourier transformation (see lines 10-11 in Algorithm 1, see Fig. 2b).

The very classical approach in finding eigenfrequencies is to look for local maxima of the eigenmode spectrum. However, this way is not efficient when the interest is in precise determination of the resonant frequencies and has some drawbacks. Firstly, the spectrum is discrete with a certain resolution and the peak value may not be entirely located on a sample point. The eigenfrequency could be a value that is somewhere in the range between two samples given with the frequency resolution for certain discrete frequency values ("bins"), implying that the local maximum is not always the frequency that is sought. Secondly, a more serious focus was placed that the neighboring modes contribute a certain amount to the total response at the resonance of the mode being analyzed and affect slightly the resonant frequency. To deal with these problems, refined modal extraction methods based on signal processing techniques have been developed (see lines 12-31 in Algorithm 1).

\subsubsection{Technique for locating a Gaussian pulse}

For further analysis, local Gaussian pulses (see Fig. 2c) within the spectrum should be located properly (see line 13 in Algorithm 1). The location process is divided into several steps. Primarily, a local Gaussian pulse is found as a set of samples with a local maximum. Thereafter, supplementary check is conducted if some other samples might be added to the right-most/left-most side of the current Gaussian pulse. Namely, if the average amplitude of the succeeding right/left outer triple of samples is less than the average amplitude of the right-most/left-most triple of samples, the outer triple of samples is added appropriately to the right/left part of the Gaussian pulse. After that, the "empirical rule" for the current located Gaussian pulse is applied. For this purpose, the standard deviation for the pulse is estimated and four standard deviations are accounted for the resulting pulse. At the end, the distance from the both ends of the Gaussian pulse to its maximum is equally adjusted.

\subsubsection{Fitting models}

By invoking the acquired knowledge of the eigenmode spectrum of the cavity, the fitting model should be nonlinear in the parameters, i.e. we should use Gaussian nonlinear model to obtain the parameters. In this direction, the parametric fitting is involved as essential technique for precise determination of the eigenfrequencies and reducing the amount of data required for a given resolution. Relying on the above discussion, a custom Gaussian model is modeled within the MATLAB curve fitting tool (see line 16 in Algorithm 1), which suits to the specific curve fitting needs, as shown below:

$y_{i}=a_{i} \epsilon^{\frac{-\left(f-f_{i}\right)^{2}}{2 \sigma_{i}^{2}}}$,

where $i$ goes from unity to the number of the located local Gaussian pulses in the amplitude spectrum for the analyzed structure. With this model all of the local Gaussian pulses are fitted and in each $i$-th fitting the values for the found parameters $a_{i}, f_{i}$, and $\sigma_{i}$ are saved. The parameters $a_{i}$ and $f_{i}$ are used as initial reasonable starting values of the parameters in the further fit, whereas the parameter $\sigma_{i}$ is included to increase the numerical robustness. The values of the parameter $f_{i}$ are good candidates for eigenfrequencies, since they give the position of the maximum value of each Gaussian pulse.

However, following this way we are restricted to a limited number of samples, same as the number of samples which constitute the local Gaussian pulse. The limitation in the samples causes that the coefficient, which represents the goodness of fitting, is very low. That means that the accuracy of the eventual eigenfrequency cannot be high. Additionally, with this approach only the amplitude information of the signal is used, which is not sufficient for precise extraction.

These disadvantages lead to extending our approach, in a sense where we can get also the phase information of the signal in a form that is suitable for implementation. Namely, after fitting a local Gaussian pulse in frequency domain the values of the parameters and the coefficients for the goodness of the fit are obtained. If the goodness of the fit for some Gaussian pulse is positive then we window this Gaussian pulse with Gaussian windowing function and we go back in time domain using Inverse Fast Fourier Transform (IFFT) (see lines 18-31 in Algorithm 1). Otherwise, we do not take into consideration this pulse, which is most probably noise. From signal processing is known that shifting in frequency domain means modulation in time domain. So, the Gaussianmodulated sinusoidal signal $y_{i}=a_{i} \sin \left(2 \pi f_{i} t-\phi_{i}\right) \epsilon^{\frac{-\left(t-t_{0}\right)^{2}}{2 \sigma_{i}^{2}}}$ is expected in time domain. The frequency of the modulation $f_{i}$ is exactly the frequency that is sought. Consequently, we fit the resulting signal in time domain with a custom Gaussian modulated model of the cavity response and again by finding the optimal model parameters in the least squares sense we determine the "true" eigenfrequency. 
The main advantage comparing the proposed approach with the classical approach for finding the peaks, is that within this approach the phase information of the signal is used and parametric fitting with all of the data available in the time domain representation is applied. Although, the fitting based only on the amplitude information of the signal results in poor fit, now using the phase information of the signal, very good value for the goodness of the fit can be reached. In this way, as shown in Sect. 3, we gain a very high accuracy in the eigenfrequency extraction from time domain computations.

\subsubsection{Nonlinear parametric fitting}

The fitting process uses the method of nonlinear least squares, presented in Nash and Sofer (1996) and Lewis et al. (2006), which minimizes the summed square of residuals, identified as error associated with the data, given by

$S=\sum_{i=1}^{n} r_{i}^{2}=\sum_{i=1}^{n}\left(y_{i}-\hat{y}_{i}\right)^{2}$,

where the residual $r_{i}$ for the $i$-th data point is defined as the difference between the observed response value $y_{i}$ and the fitted response value $\hat{y}_{i}$ while $n$ is the number of data points included in the fit. In case of nonlinear models the parameters cannot be estimated using simple matrix techniques and these models are particularly sensitive to the starting points. This leads to difficult fit and the starting points are adjusted. Therefore, at the beginning initial reasonable starting values for each parameter are provided. Then, the iterative approach follows some steps until the fit reaches the specified convergence criteria. At the end, the direction and magnitude of the adjustment of the parameters depends on the LevenbergMarquardt fitting algorithm proposed by Levenberg (1944), Marquardt (1963), and Moré (1978).

\section{Simulation results}

The accuracy of the TD method for eigenfrequency extraction is tested for both analytically and non-analytically resolvable problems. Additionally, the proper functionality of the method has been checked during its implementation process. In the numerical tests, several resonators are considered. Namely, due to verification purposes, rectangular, cylindrical and spherical resonant structures are analyzed, whose exact solution can be analytically evaluated. Firstly, the eigenvalues of the above mentioned resonators are computed from their analytical expressions and following this way, a logarithmic relative error is calculated as

relative error $=\log _{10} \frac{\left|f_{\text {analytical }}-f_{\text {numerical }}\right|}{f_{\text {analytical }}}$,

by considering the first computed mode eigenfrequency, unless otherwise stated.
Besides the analytically resolvable resonators, the relative error is also measured for the chaotic billiard resonator, which has a clustered eigenvalue distribution (Dembowski et al., 2002). A clustered distribution containing too close eigenfrequencies might cause difficulties in the eigenfrequency extraction. As reference data, eigenvalues calculated with CEM3D eigenmode solver for different degrees of freedom (DOF) are computed. Here, CEM3D eigenmode solver is a parallel program, developed by (Ackermann and Weiland, 2012) for the accurate calculations of eigenfrequencies for a given structure. It implements a FEM formulation by means of higher order curvilinear elements.

In all simulations, we first modeled and meshed the related geometries in CST MWS with hexahedrons and saved the electric field intensity during the transient simulation. Afterward, the eigenvalue extraction is calculated in MATLAB.

In the simulation studies, we experienced that for the time domain field computations, a single personal computer is suited for problems with a moderate number of degrees of freedom (say, up to $10^{6} \mathrm{DOF}$ ). To be precise, a computer with a single core Pentium $3 \mathrm{GHz}$ processor and $6 \mathrm{~GB}$ main memory was used. The same PC configuration was also utilized for the TD method for eigenvalue extraction. On the other hand, a more powerful computer was necessarily used to enable the handling of meshes with hundreds of thousands up to several millions DOF. The larger-scale simulations were performed on a GPU computer, e.g. $2.00 \mathrm{GHz}$ (quadcore) having 32 GB RAM memory and 4 nVIDIA Quadro GPUs.

\subsection{Rectangular, cylindrical, and spherical cavity}

As a first experiment, we consider a rectangular cavity with perfectly conducting walls, containing a perfect vacuum. The degeneracy is broken by making the side lengths different, i.e. rectangular resonator with dimensions $a=20 \mathrm{~cm}, b=$ $10 e \mathrm{~cm}$, and $c=10 \pi \mathrm{cm}$. The resonance frequency of the rectangular microwave cavity for the $\mathrm{TE}_{011}$ mode (the mode with the lowest cutoff frequency for a rectangular waveguide where $c>b>a$ ) is found by imposing boundary conditions on the electromagnetic field expressions in which all of the field components vary sinusoidally at a single frequency (Pozar, 1998).

Along this line, the fundamental mode in a cylindrical cavity (Reitz and Milford, 1960) with radius $R=20 \mathrm{~cm}$ and length $L=10 \pi \mathrm{cm}$ is calculated, too. For the analyzed cylindrical cavity, since we do not have large $L$ fulfilling $L>$ $2.03 R$, the $\mathrm{TM}_{010}$ mode constitutes the fundamental oscillation. The mode of interest has azimuthal symmetry and the electric field has no longitudinal variation $(\delta E / \delta z=0)$.

Lastly, the first $\mathrm{TM}_{101}$ mode of a spherical resonator with a radius $R=1 \mathrm{~m}$ is computed from analytical expressions given in (Gallagher and Gallagher, 1985) by employing a root finding algorithm of transcendental equations which is simply explained in (Pozar, 1998). 


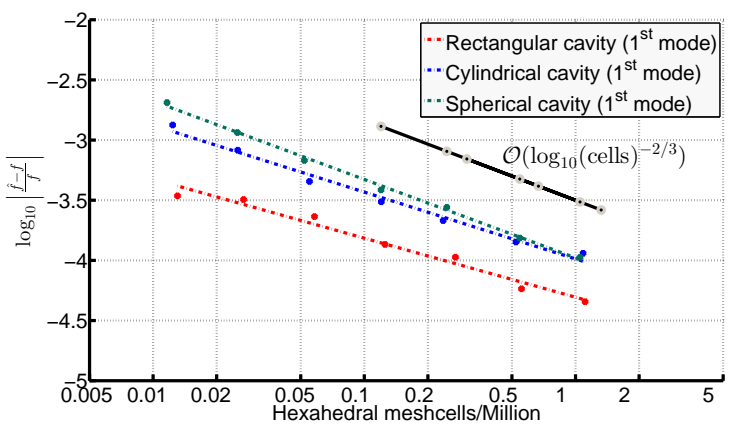

Fig. 3. Relative deviation of the numerically obtained values $\hat{f}$ for the lowest eigenfrequency to the analytical result $f$ as a function of the degrees of freedom (DOF) for a rectangular, cylindrical, and spherical resonator.

Specifically, for the time domain field simulations several different discretization meshes have been used and the convergence study based on the calculation of a relative error, given with Eq. (3), is shown in Fig. 3. As the number of discretization mesh cells increases, the difference between the analytical and the numerical solutions becomes smaller and absolute error in order of $10^{-4}$ is present. So, fast convergence is observable and it should be emphasized. As suggested by the convergence study, good accordance of the numerical with the analytical results is evident.

\subsection{Billiard cavity}

Using the previosly described algorithm, about 900 eigenfrequencies up to $7 \mathrm{GHz}$ have been calculated for the billiard cavity structure. Unfortunately, for this shape of resonator an analytical solution for the electromagnetic problem is not available and in order to validate the obtained results, reference data from CEM3D eigenmode solver are used (see Fig. 4). The structures are modeled and meshed with curvilinear tetrahedrons and the corresponding meshes are imported to CEM3D eigenvalue solver in order to compute the requested eigenfrequencies for the analyzed structure.

In Fig. 4, a part of the results that are found with the TD approach compared to the reference data, is presented. On the abscissa are given the frequencies in an a priori selected frequency band, i.e. from 1.8 up to $2.4 \mathrm{GHz}$. The ordinate shows the eigenfrequencies obtained with the time domain approach using four different discretization meshes, and the reference data calculated using a field simulation in frequency domain with two different tetrahedral meshes. The total time for the transient simulations is $3.5 \times 10^{-5} \mathrm{~s}$, which results in frequency resolution of $30 \mathrm{kHz}$.

The results in Fig. 4 indicate that when fine mesh is used the number of eigenfrequencies found with the proposed TD approach is exactly the same as the reference data, i.e. no additional frequency is added or no missed. In addition, such check was conducted for all of the 900 calculated eigen-

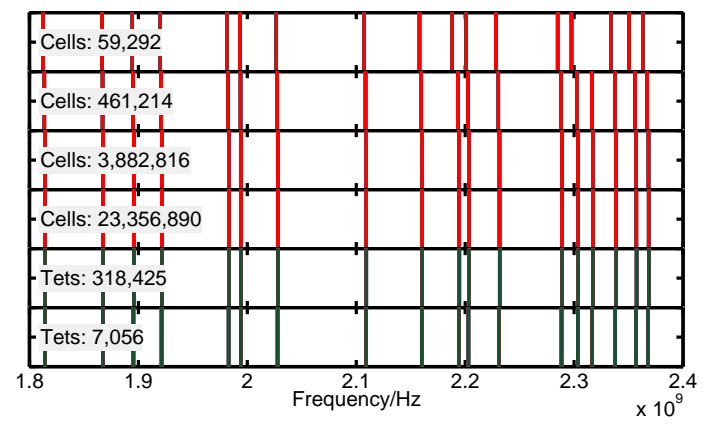

Fig. 4. Convergence study showing a comparison between the eigenfrequencies calculated with the proposed TD approach (red color) and the reference eigenfrequencies obtained with CEM3D eigenmode solver based on higher order curvilinear elements (green color). For the TD approach four different hexahedron discretization meshes are used. At the same time, the reference data are obtained using two different tetrahedral discretization meshes.

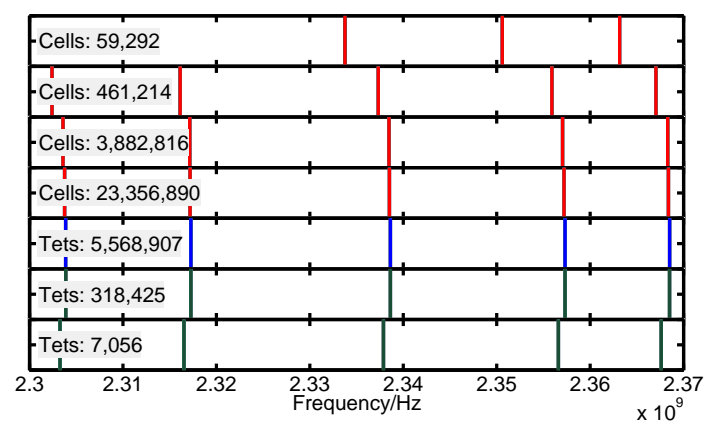

Fig. 5. Convergence study showing a comparison between the eigenfrequencies calculated with the proposed TD approach (red color) and the reference eigenfrequencies obtained with CEM3D eigenmode solver based on higher order curvilinear elements (green and blue color). For the TD approach four different hexahedron discretization meshes are used. At the same time, the reference data are obtained using three different tetrahedral discretization meshes.

frequencies. Concerning the accuracy of the obtained data, slightly shifting of the frequencies can be observed in case of coarse meshes. As the number of mesh cells increases, a good agreement with the reference data is clearly observed. For this purpose, a part of Fig. 4 is enlarged and shown in Fig. 5. According to Fig. 5, in case of fine meshes a good agreement of TD results with the reference data can be obtained. Furthermore, in Fig. 5 an additional row is added representing extremely accurate reference data for the eigenvalues in the range [2.30, 2.37] GHz. These results are obtained with almost 6 million of tetrahedral mesh cells and are used to calculate the logarithmic relative error for the modes: 2.36 and $2.37 \mathrm{GHz}$ (see Fig. 6). From this figure a second order convergence error is observed. Consequently, the proposed 


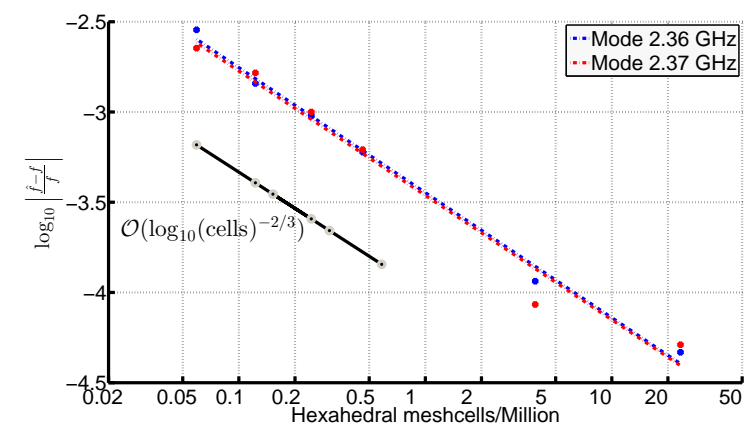

Fig. 6. Relative deviation of the numerically obtained values $\hat{f}$ to the reference results $f$ as a function of the degrees of freedom (DOF) for a billiard resonator. Two eigenfrequencies are considered: $2.36 \mathrm{GHz}$ and $2.37 \mathrm{GHz}$ mode eigenfrequency.

approach can be used for precise extraction of eigenfrequencies from time domain computations.

\section{Conclusions}

In conclusion, we have presented an approach for eigenfrequency extraction when the time domain response for a superconducting closed resonator is available and when plenty of eigenfrequencies are required. The approach is relatively fast and very simple compared to others presented in literature. In addition, we have inspected the robustness and convergence of the proposed technique in time domain with the reference data determined by the CEM3D eigenmode solver with higher order curvilinear elements. Hereby, the conclusion shows that the proposed approach results in solutions which agree well with the reference data, gaining high accuracy and efficiency in eigenvalue extraction. Finally, all of the results indicate that the proposed TD technique can be used in different areas of applications, where a precise extraction of plenty of eigenfrequencies takes a crucial role.
Acknowledgements. The work of Todorka Banova is supported by the "Excellence Initiative" of the German Federal and State Governments and the Graduate School of Computational Engineering at Technische Universität Darmstadt.

\section{References}

Ackermann, W. and Weiland, T.: High Precision Cavity Simulations, Proceedings of the 11th International Computational Accelerator Physics Conference, 1-5, 2012.

CST AG, CST 2012, Darmstadt, Germany, MICROWAVE STUDIO, 2012.

Dembowski, C., Dietz, B., Gräf, H.D., Heine, A., Papenbrock, T., Richter, A., and Richter, C.: Experimental Test of a Trace Formula for a Chaotic Three Dimensional Microwave Cavity, Phys. Rev. Lett., 89, 064101, doi:10.1103/PhysRevLett.89.064101, 2002.

Dembowski, C., Dietz, B., Friedrich, T., Gräf, H.D., Harney, H.L., Heine, A., Miski-Oglu, M., and Richter, A.: Distribution of resonance strengths in microwave billiards of mixed and chaotic dynamics, Phys. Rev. E, 71, 046202, doi:10.1103/PhysRevE.71.046202, 2005.

Gallagher, S. and Gallagher, W. J.: The Spherical Resonator, IEEE Trans. on Nuclear Sci. Vol NS-32 5, 1985.

Mandal, M. and Asif, A.: Continuous and Discrete Time Signals and Systems, Posts \& Telecom Press, 2010.

Marquardt, D.: An Algorithm for Least Squares Estimation of Nonlinear Parameters, SIAM J. Appl. Math, 11, 431-441, 1963.

MATLAB R2011b, The MathWorks Inc., Natick, MA, 2011.

Moré, J. J.: The Levenberg-Marquardt Algorithm: Implementation and Theory, Lecture Notes in Mathematics, v630, edited by: Watson, G., Springer, 1978.

Nash, S. G. and Sofer, A.: Linear and Nonlinear Programming, McGraw-Hill Companies, Inc, New York, ISBN 0-07-046065-5, 1996.

Levenberg, K.: A Method for the Solution of Certain Problems in Least Squares, Quart. Appl. Math, 2, 164-168, 1944.

Lewis, J. M., Lakshmivarahan, S., and Dhall, S.: Dynamic Data Assimilation: A Least Squares Approach (Encyclopedia of Mathematics and its Applications), Cambridge University Press, 2006.

Pozar, D. M.: Microwave Engineering, 2nd edition, John Wiley and Sons, Inc., New York, NY, 1998.

Reitz, J. R. and Milford, F. J.: Foundations of Electromagnetic Theory, Addison-Wesley, 1960.

Saad, Y.: Numerical Methods for Large Eigenvalue Problems, Revised Edition, Society for Industrial and Applied Mathematics, 2011. 